ORIGINAL ARTICLE

\title{
Culture confirmed multidrug resistant tuberculosis: diagnostic delay, clinical features, and outcome
}

\author{
H S Schaaf, K Shean, P R Donald
}

\begin{abstract}
See end of article for authors' affiliations .....................

Correspondence to: Dr H S Schaaf, Department of Paediatrics and Child Health, Faculty of Health Sciences, Stellenbosch University, PO Box 19063, Tygerberg 7505, South Africa; hss@sun.ac.za
\end{abstract}

Accepted 2 May 2003

\begin{abstract}
Aims: To determine the delay in diagnosis of multidrug resistant (MDR) tuberculosis (TB), the correlation between drug susceptibility patterns of adult-child contact pairs, the effectiveness of treatment, and the outcome in these children.

Methods: MDR M tuberculosis culture results of children were prospectively collected during a four year period in the Western Cape Province of South Africa, an area with a TB incidence of 589/100 000 population, and a new MDR TB rate of $0.94 \%$. Folder reviews were done to retrieve clinical information. Children not already on treatment at our MDR TB clinic or TB hospital were recalled and appropriate treatment was started. Follow up was done for as long as possible.

Results: Thirty nine children, median age 4.5 years at first TB diagnosis and 6.2 years on MDR culture confirmation, were seen. Delay in starting appropriate MDR treatment after TB diagnosis was a median of 2 days if MDR TB source cases were taken into account, but 246 days if the drug susceptibility pattern of the source case was not considered, and 283 days if there was no known tuberculosis source case. Correlation between the drug susceptibility results of the child's and adult source case's isolates was $68 \%$. Seventeen children had smear positive tuberculosis, of whom 13 had cavitatory pulmonary disease. Eight children had central nervous system TB. Thirty six children were treated for MDR tuberculosis, of whom four died.

Conclusions: Obtaining a detailed contact history is essential as a delay in starting appropriate MDR antituberculosis treatment has potentially serious consequences.
\end{abstract}

$M$ ultidrug resistant (MDR) tuberculosis (TB) has important implications for individual patients and tuberculosis control programmes. Although the management of MDR TB in adults has been extensively documented, ${ }^{1-3}$ relatively little is known about the diagnosis and management of childhood MDR TB.

Adult patients develop both new (previously "primary" resistance-that is, no previous antituberculosis treatment) or previously treated (previously "acquired" resistance-that is, after previous antituberculosis treatment) MDR TB. In a high TB incidence area, Van Rie and colleagues ${ }^{4}$ have, however, shown by restriction fragment length polymorphism (RFLP) analysis that, even in some cases of previously treated MDR TB, the MDR Mycobacterium tuberculosis strain was actually transmitted. In children, tuberculosis is usually paucibacillary, which makes the development of drug resistance, and especially MDR TB through treatment, less likely. Because of their age, recent transmission of infection is usually assumed; it has been documented that transmission of MDR TB in children occurs, ${ }^{5}$ and furthermore, is as likely to occur as is the case with drug susceptible organisms. ${ }^{67}$ Nonetheless it is also known that many children in high incidence areas will have received previous antituberculosis treatment or chemoprophylaxis. ${ }^{8}$

Tuberculosis morbidity and mortality rates remain relatively high in children, and delay in diagnosis and effective treatment can further increase these rates because of disease progression and development of complications such as disseminated TB and tuberculous meningitis (TBM). Delay in diagnosis and treatment of tuberculosis can be due to patient delay or doctor delay; for example, late presentation with symptoms or failure of the doctor to consider the diagnosis of tuberculosis. " In the case of drug resistant tuberculosis this delay in initiating effective treatment can be even longer, especially if the attending physician does not consider the diagnosis, but also because of the time required for culture of the organism and determination of drug susceptibility (that is, laboratory delay).

The clinical management of MDR TB in children is not well documented. Some basic principles that apply to the management of adult MDR TB cases, such as not adding a single drug to a failing regimen and giving at least two or preferably three drugs to which the patient's $M$ tuberculosis isolate is susceptible, probably hold true for childhood MDR TB. ${ }^{10}$ However, the paucibacillary nature of childhood tuberculosis may imply that fewer drugs and a shorter treatment duration will be sufficient, but no prospective studies are available to confirm this. ${ }^{7}$ Drugs used in the treatment of MDR TB are generally more toxic than first line drugs; some drugs, such as the fluoroquinolones, are not generally recommended for use in children, ${ }^{11}$ even though their safety in short and medium term treatment has been documented. ${ }^{12}$

Outcome of treatment of adult MDR TB cases has been relatively poor until recently, with very high mortality rates. $^{13}{ }^{14}$ Little is known about the long term outcome of childhood MDR TB cases.

The aim of this study was to determine the duration of, and the reasons for, the delay in initiating appropriate antituberculosis treatment in children with MDR TB. The further aims were to compare the drug susceptibility patterns of adult-child contact pairs and to describe the clinical features,

Abbreviations: AFB acid fast bacilli; DOT directly observed treatment: DSP, drug susceptibility pattern; INH, isoniazid; MDR, multidrug resistant; RFLP, restriction fragment length polymorphism; RMP, rifampicin; TB, tuberculosis; TBM, tuberculous meningitis 
treatment, and outcome of children with culture confirmed MDR TB.

\section{PATIENTS AND METHODS Setting}

All multidrug resistant (defined as cultures resistant to isoniazid (INH) and rifampicin (RMP), with or without resistance to other antituberculosis drugs) $M$ tuberculosis culture results from children (defined as patients $<15$ years of age) in two of the four regions of the Western Cape Province of South Africa, the Cape Town Metropole, and Westcoast/Winelands regions, were prospectively collected for the period 1 January 1998 to 31 December 2001. These two regions contained $77.6 \%$ of the total population of 4.384 million for this province in 2002 based on the 1996 national census (Department of Health: Directorate Health Systems Research and Epidemiology).

This province had a reported TB notification rate of 589 new cases per 100000 population per year in 1998 (Department of Health: Directorate Health Systems Research and Epidemiology). The rate of new and previously treated multidrug resistance determined in adult TB cases in the Western Cape Province during 2001-02 was 0.94\% (95\% confidence interval (CI) $0.16 \%$ to $3.70 \%$ ) and $3.95 \%$ (95\% CI $1.37 \%$ to $9.56 \%$ ) respectively (unpublished data, Weyer K et al, South African Medical Research Council). MDR TB was found in seven $(2.3 \%)$ of 306 children $<13$ years of age with culture confirmed $\mathrm{TB}$ in a referral hospital in the same province during 1994 to $1998 .{ }^{15}$ Prevalence of human immunodeficiency virus (HIV) infection in women attending antenatal clinics in the Western Cape Province rose from $5.2 \%$ (95\% CI $4.2 \%$ to $7.2 \%$ ) in 1998 to $8.6 \%$ (95\% CI $5.6 \%$ to $11.6 \%$ ) in 2001 (Department of Health: Directorate Health Systems Research and Epidemiology).

\section{Drug susceptibility testing}

Isolates of $M$ tuberculosis were sent to the South African Institute for Medical Research in Cape Town for susceptibility testing. Initial screening was done for INH and RMP resistance only. When resistance to INH and RMP was found, susceptibility testing for streptomycin, ethambutol, ethionamide, and other second line antituberculosis agents was performed.

Laboratory procedures for determining drug resistance were as follows: Middlebrook 7H12 (Bactec) culture medium was used for selective primary isolation of mycobacterial strains. The niacin production test (or polymerase chain reaction in some cases) was used to identify $M$ tuberculosis. Drug susceptibility testing was performed by the economic variant of the indirect proportion method. ${ }^{8}$ The following drugs were tested at the indicated concentrations: INH $0.2 \mu \mathrm{g} / \mathrm{ml} \mathrm{LJ}$; RMP $30 \mu \mathrm{g} / \mathrm{ml} \mathrm{LJ}$; streptomycin $5 \mu \mathrm{g} / \mathrm{ml} \mathrm{LJ}$; and ethambutol $15 \mu \mathrm{g} / \mathrm{ml} \mathrm{LJ}$, ethionamide $10 \mu \mathrm{g} / \mathrm{ml} \mathrm{LJ}$, kanamycin $5 \mu \mathrm{g} / \mathrm{ml} \mathrm{LJ}$, amikacin $40 \mu \mathrm{g} / \mathrm{ml} \mathrm{LJ}$, thiacetazone $2 \mu \mathrm{g} / \mathrm{ml} \mathrm{LJ}$, and ofloxacin $2 \mu \mathrm{g} / \mathrm{ml} \mathrm{LJ}$. The susceptibility of a strain was judged by determining the proportion of bacilli resistant to a specific drug in comparison with growth on a specific control, using international criteria. Resistance was defined as $1 \%$ or more bacterial growth. Quality assurance for drug susceptibility results is done locally with every batch and quarterly by the national tuberculosis reference laboratory. ${ }^{8}$

\section{Clinical data}

The clinical records of all children with positive cultures for MDR $M$ tuberculosis were reviewed. If children were not already prospectively known to us either by follow up at our MDR tuberculosis clinic or admitted to our local TB hospital, patients were recalled and special efforts, such as home visits, were implemented to trace them. In addition to recorded information a history was obtained at the time of the study from all children and/or their caregivers regarding previous TB chemoprophylaxis or treatment, and whether they had close contact with adults with pulmonary TB. Culture and susceptibility results from identified adult source cases were obtained. Contact history and culture information was verified with personnel at the local authority health clinics. History of recent weight loss or failure to gain in weight, cough for more than two weeks, fever, or other relevant symptoms were documented.

Weight at diagnosis and site of tuberculosis was recorded. Results of tuberculin skin test (Mantoux test by intradermal injection, 5 tuberculin units, Japanese purified protein derivative), HIV serology, and sites from which $M$ tuberculosis was cultured were noted where available. A transverse diameter of induration of $\geqslant 15 \mathrm{~mm}$ ( $\geqslant 5 \mathrm{~mm}$ for HIV infected children) after Mantoux skin testing was regarded as significantly positive in accordance with World Health Organisation (WHO) criteria, as more than $90 \%$ of children in this area receive BCG. ${ }^{16}$ Chest radiographs were read according to a standardised method. ${ }^{17}$

Treatment delay was calculated as the number of days from the time that MDR TB disease should, in retrospect, have clinically been suspected until appropriate treatment for MDR TB was initiated. Children were treated either according to the drug susceptibility pattern (DSP) of the adult source case if treatment was started before the child's isolate was available and the child had a known source case, or according to the child's own isolate's DSP. Two or more drugs were used to which the organism was susceptible. Duration of treatment prescribed was from 9 to 12 months after the last positive culture depending on the severity of disease. All children were followed up clinically and radiologically for as long as possible and follow up cultures were done during and towards the end of treatment to confirm cure wherever possible. Outcome by the end of the study was documented.

All treatment was given as directly observed therapy, either in hospital or at the local clinic. Children had to attend or caregivers were expected to take the children to the local health clinic 5 days a week where health care workers had to observe the children taking their treatment. In some cases community volunteers were employed to directly observe treatment. When patients did not return for treatment, several home visits were made to motivate caregivers to bring the children for treatment. If despite all efforts (including motivation for hospital admission for treatment) children did not receive any treatment for more than two months, treatment was discontinued. Follow up visits were, however, continued at our MDR clinic.

The study was approved by the Institutional Review Board of the Faculty of Health Sciences of Stellenbosch University.

\section{RESULTS}

\section{Clinical and demographic data}

MDR $M$ tuberculosis isolates were obtained from 39 children ( 20 boys and 19 girls), during the four year study period. The median age at first diagnosis of tuberculosis was 4.5 years (range 0.4-14.9 years), but median age at which MDR tuberculosis was eventually confirmed by culture was 6.2 years (range 0.4-16.2 years). Table 1 summarises the age at which MDR TB disease was clinically suspected, the clinical features, chest radiograph results, and type of TB.

Twenty seven (69\%) children had a history of contact with one or more adults with pulmonary tuberculosis. Of these, 21 (54\%) children had contact with one or more known cases of MDR TB, one child had contact with an isoniazid and streptomycin resistant source case, four children had drug susceptible source cases, and in four children the source 
Table 1 Age, clinical features, and chest radiograph results of children with MDR TB

\begin{tabular}{|c|c|}
\hline Clinical feature & No. $(\%)(n=39)$ \\
\hline \multicolumn{2}{|l|}{ Age MDR TB presented most likely } \\
\hline 0 to $<5$ years & $19(49)$ \\
\hline 5 to $<10$ years & $7(18)$ \\
\hline 10 to $<15$ years & $13(33)$ \\
\hline \multicolumn{2}{|l|}{ Common presenting symptoms } \\
\hline Loss of weight or failure to gain weight & 29 (74) \\
\hline Cough $>2$ weeks & $25(64)$ \\
\hline Fever & $16(41)$ \\
\hline Convulsions & $7(18)$ \\
\hline Lymphadenopathy or abscess & $5(13)$ \\
\hline \multicolumn{2}{|l|}{ Mantoux skin test result } \\
\hline$\geqslant 15 \mathrm{~mm}$ induration & $18 / 22(82)$ \\
\hline$\geqslant 10$ to $14 \mathrm{~mm}$ induration & $1 / 22(4)$ \\
\hline 0 to $4 \mathrm{~mm}$ induration & $3 / 22(14)$ \\
\hline \multicolumn{2}{|l|}{ HIV status } \\
\hline Seronegative & $23(59)$ \\
\hline Seropositive & $6(15)$ \\
\hline Not tested & $10(26)$ \\
\hline Pulmonary tuberculosis & $37(95)$ \\
\hline Primary & $26(67)$ \\
\hline Adult type & $11(28)$ \\
\hline Extrapulmonary tuberculosis & $18(46)^{*}$ \\
\hline Tuberculous meningitis (3) or granuloma brain (5) & $8(21)$ \\
\hline Miliary TB & $2(5)$ \\
\hline Peripheral lymph node/abscess confirmed TB & $5(13)$ \\
\hline Abdominal TB & $4(10)$ \\
\hline Pleural effusion & $4(10)$ \\
\hline Bone TB & $2(5)$ \\
\hline Otitis media & $1(3)$ \\
\hline \multicolumn{2}{|l|}{ Chest radiograph results $†$} \\
\hline Hilar/mediastinal lymphadenopathy & $19(51)$ \\
\hline Large airways compression & $5(14)$ \\
\hline Segmental/lobar opacification & $26(70)$ \\
\hline Cavities & $14(38)$ \\
\hline Bronchopneumonic opacification & $7(19)$ \\
\hline Pleural effusion & $4(11)$ \\
\hline Miliary & $2(5)$ \\
\hline Normal & $1(3)$ \\
\hline
\end{tabular}

cases' drug susceptibility patterns were unknown. Three children had contact with both drug resistant and other source cases.

Table 2 summarises the reasons for the delay in diagnosis and initiating appropriate treatment for MDR $\mathrm{TB}$ in the children.

\section{Smear microscopy and drug susceptibility results}

Smear microscopy for acid fast bacilli (AFB) was done on sputum and/or gastric aspirate specimens from 34 (87\%) children. Of these, 17 children had smear positive pulmonary tuberculosis, of whom 13 had cavities on their chest
Table 3 Resistance of $M$ tuberculosis strains to drugs in addition to INH and RMP

\begin{tabular}{ll}
\hline Antituberculosis drug & No. $(\%)(\mathbf{n}=37)$ \\
\hline Streptomycin & $19(49)$ \\
Ethambutol & $4(10)$ \\
Ethionamide & $7(18)$ \\
Kanamycin/amikacin & 2 of $35(6)$ \\
Thiacetazone & 4 of $35(11)$ \\
Ofloxacin & 2 of $30(7)$ \\
Cycloserine & 1 of $8(12)$ \\
Pyrazinamide* & 1 of 1 \\
\hline *Susceptibility rarely tested. &
\end{tabular}

radiographs. The children with smear positive disease tended to be older, with $4 / 19(21 \%)$ from 0 to $<5$ years of age, $4 / 7$ $(57 \%) 5$ to $<10$ years of age, and $9 / 13(69 \%)$ in the age group 10 to $<15$ years of age $(\mathrm{p}=0.019)$.

The median number of drugs to which the children's $M$ tuberculosis strains were resistant was 3 (range 2-6). Table 3 summarises resistance to drugs in addition to INH and RMP.

Drug susceptibility patterns from adult drug resistant source cases' isolates $(n=22)$ were identical to those of the child contacts in $15(68 \%)$ cases, of which two were additionally confirmed by RFLP analysis. ${ }^{5}$ In a further three (13\%) cases, only thiacetazone resistance was found in addition to the child isolate's DSP (2) or the child's strain was additionally resistant to ethionamid (1). In four children the source cases' DSPs were not the same as those from the children, but in one of these cases RFLP analysis was done which confirmed the mother's strain to be the same as one of two strains with which the child was infected. In the remaining child, the DSP of the $M$ tuberculosis isolate was incomplete and could not be compared with the source case's strain.

\section{Previous antituberculosis treatment}

Seventeen children received isoniazid and rifampicin based antituberculosis treatment for three or more months before the diagnosis of MDR TB was made. Only one child with extensive lymphobronchial disease completed a three month course of chemoprophylaxis (isoniazid and rifampicin), but despite compliant treatment developed MDR TB three months later.

Six children received previous treatment for laboratory confirmed drug susceptible tuberculosis. Of these, three had known contact only with MDR TB household source cases at the time of first presentation. Response to compliant isoniazid-rifampicin based treatment was poor in all three cases and MDR TB was confirmed 2, 11, and 41 months after completion of drug susceptible therapy, respectively. The last

\begin{tabular}{|c|c|c|}
\hline Manner of identification and/or reason for delay* & No. (\%) & $\begin{array}{l}\text { Delay in days, median } \\
\text { (range) }\end{array}$ \\
\hline \multicolumn{3}{|l|}{ Household MDR TB source cases known at time of diagnosis } \\
\hline MDR TB treatment from TB diagnosis & $9(23)$ & $2(0-45)$ \\
\hline Child not managed as MDR contact & $11(28 \%)$ & 246 (77-1537) \\
\hline Community contact MDR identified after MDR culture result & $2(5)$ & \\
\hline Response to culture/susceptibility result only (2 MDR contacts) & $10(26)$ & $77(37-176)$ \\
\hline \multicolumn{3}{|l|}{$\begin{array}{l}\text { Poor response (deterioration or relapse) despite treatment for drug } \\
\text { susceptible TB (including isoniazid and rifampicin) }\end{array}$} \\
\hline Initial MDR result disregarded & $3(8)$ & $294(281-590)$ \\
\hline Culture/susceptibility done in response to deterioration & $11(28)$ & $263(103-1537)$ \\
\hline $\begin{array}{l}\text { MDR confirmed culture, but no MDR treatment [untraceable (2) or } \\
\text { clinically well when traced (1)] }\end{array}$ & $3(8)$ & \\
\hline
\end{tabular}


child died soon after MDR TB was diagnosed because of extensive bilateral lung destruction. In the first child, the RFLP analyses of the child's and mother's $M$ tuberculosis strains were identical, the mother having had MDR TB for several years.

Two of the children initially infected with drug susceptible strains, were most likely reinfected with MDR strains. Both were HIV infected. In each child, susceptibility tests were done on two separate specimens during the first episode of tuberculosis, both of which were susceptible to isoniazid and rifampicin. In one case, the mother, who first had drug susceptible TB, developed MDR TB during the course of her illness, while the child was treated and cured in hospital from his first TB episode. He then developed MDR TB, the resistant organism being cultured from gastric aspirates and an ear swab 22 months later while staying with his mother and aunt, the latter having also developed new MDR TB. He died soon thereafter of advanced HIV disease and TB. The second child had no identified TB source cases. He received directly observed treatment (DOT) in hospital for six months for the first episode of drug susceptible TB and was discharged cured with negative cultures. He returned 19 months after completing treatment with MDR TB, confirmed on several specimens, and was treated for MDR TB with DOT for 18 months. He subsequently returned with a third episode of TB 18 months after completing the second course of antituberculosis treatment, again with a drug susceptible strain. He was started on isoniazid-rifampicin based DOT and responded well to treatment.

The remaining child that initially had drug susceptible TB presented with adult type cavitating pulmonary TB. Both parents had confirmed drug susceptible TB. She most likely acquired MDR TB, as she was a severe defaulter, often noted to spit out her treatment, and eventually developed MDR TB 11 months into repeatedly interrupted and non-compliant treatment.

A further nine children received a course of isoniazidrifampicin based antituberculosis treatment, duration ranging from 4 to 30 months, with poor response to treatment or relapse of $\mathrm{TB}$ in all nine cases with no initial cultures or susceptibility tests done. In five of these children, contact with adult MDR cases in the same household was evident. One child developed miliary TB and tuberculous meningitis despite short course treatment, while there was a history of contact with several adult cases of MDR TB. In the four children who had no known contact with MDR TB cases, drug resistance could have been acquired, but compliance with treatment was good in all but one case and the response to treatment was poor throughout the course of treatment.

The remaining child of the 17 who received previous treatment had primary TB at 1 year of age. She was treated and cured, but 12 years later developed MDR TB, most likely from contact with several adult household MDR TB cases.

\section{Management of MDR TB cases}

Three children received no antituberculosis drugs. Of these, two children were admitted for malnutrition and pneumonia and investigated for tuberculosis, but they were untraceable when the tuberculosis culture became available. The remaining child, who did not receive treatment, was a 13 year old girl who initially complained of pleuritic chest pain and marked weight loss. No chest radiograph was initially done, and she was traced 13 months after sputum was obtained for culture. By this time she was clinically well, her weight had returned to normal, and the chest radiograph was normal. At this point no treatment was given and only follow up was arranged.

Thirty six children received treatment for MDR TB. Two received only standard retreatment regimens, ${ }^{18}$ but for an extended duration of 9 and 18 months and were cured. All other children were treated either according to the DSP of the child's own $M$ tuberculosis strain or to the DSP of the adult index case's strain with regimens that contained two or three drugs to which the strain was susceptible. Treatment duration was aimed at 9 months for mild forms of primary $\mathrm{TB}$ and 12 months after the last positive culture for more severe cases of MDR TB. Table 4 summarises the drugs that were used, the duration of their use, and documented side effects.

Twenty three $(66 \%)$ children were hospitalised for treatment. Median hospital stay, only considering stay for MDR TB treatment, was 7 months (range 2-18 months). The remainder of the treatment for these children as well as for those not admitted was given as DOT at the local clinics.

Treatment compliance and completion for these children were as follows: $17(49 \%)$ completed treatment and were compliant; 8 (23\%) had poor compliance ( $<70 \%$ drugs taken) or interrupted treatment for $>1$ month, of whom four are still on treatment; six (17\%) defaulted before the end of treatment at between 5 and 16 months of treatment; five are still on treatment; and three children died while on treatment.

\section{Outcome of long term follow up}

Death was the outcome in four $(10 \%)$ patients. Of these, two HIV seronegative children died of MDR TB while on treatment, one because of stage $3 \mathrm{TBM}$ and the other of extensive lung involvement. Both children had known household contact with several MDR TB source cases, but opportunities for the initiation of appropriate treatment were missed. The remaining two children were HIV infected. In one child MDR TB treatment was stopped because of end stage AIDS; he died soon after. The second child was cured of MDR TB, but died nine months later because of severe acute diarrhoea and pneumonia (cultures for $M$ tuberculosis remained negative).

Twenty one (54\%) children completed treatment and were cured with cultures remaining negative. Six (15\%) children defaulted treatment after a minimum of five months treatment; four of these were lost to follow up. Remaining pathology due to TB in these two groups of children was bronchiectasis and/or destroyed lung in four children, vision loss due to TBM in one, and severe thoracic kyphosis in one. Median follow up was 14.8 months (range 0-36.7 months) for those not lost to follow up before completion of therapy. During this time, only one HIV infected child had another episode of TB, this time with a drug susceptible organism (see Previous antituberculosis treatment).

A further five (13\%) children were still on treatment by the end of the study. One was again culture positive after $>14$ months of treatment which was interrupted for two months, and one child was awaiting lobectomy for lobar bronchiectasis.

Three $(8 \%)$ children received no antituberculosis treatment at all. Of these, two were lost to follow up. The remaining girl, 13 years of age, probably had a pleural effusion when she presented. She was traced 13 months later and was clinically and radiologically normal. She remained well 18 months after initial diagnosis.

Table 5 summarises weight response and results of last available follow up chest radiographs.

\section{DISCUSSION}

The World Health Organisation, for epidemiological purposes, defines children as patients from 0 to 14 years of age. ${ }^{19}$ The incidence of tuberculosis in this age group is often underestimated because of the reliance on sputum smear or culture results. Furthermore, MDR TB has become a worldwide 
Table 4 Antituberculosis drugs used in MDR TB regimens, duration of use, and documented side effects

\begin{tabular}{|c|c|c|c|}
\hline $\begin{array}{l}\text { Drug and dose used } \\
(\mathrm{mg} / \mathrm{kg} / \text { day) }\end{array}$ & $\begin{array}{l}\text { No. of regimens } \\
(n=35)\end{array}$ & $\begin{array}{l}\text { Duration of use; median } \\
\text { (range) in months }\end{array}$ & Side effects* $\dagger$ \\
\hline Isoniazid (15-20) & 31 & $12(1.5-24)$ & \multirow{11}{*}{$\begin{array}{l}\text { Nausea }=1 \\
\text { Vomiting }=13 \text { (stopped in 6) } \\
\text { Vomiting }=4 \text { (stopped in } 2 \text { ); } \\
\text { no arthralgia }\end{array}$} \\
\hline Rifampicin (10-15) & 8 & $5.5(1.5-18)$ & \\
\hline Pyrazinamide (25-35) & 32 & $12(1.5-24)$ & \\
\hline Streptomycin (15-20) & 8 & $4(2-12)$ & \\
\hline Kanamycin/amikacin (15) & 12 & $3(1.5-10)$ & \\
\hline Ethambutol (15-25) & 31 & $14(5-31)$ & \\
\hline Ethionamide (10-20) & 31 & $10(<1-31)$ & \\
\hline Ofloxacin (10-15) & 32 & $12(1.5-24)$ & \\
\hline Thiacetazone $\neq$ (2-4) & 11 & $12(5-24)$ & \\
\hline Cycloserine (10) & 2 & $14 ; 20$ & \\
\hline Clofazamine & 1 & 20 & \\
\hline
\end{tabular}

*Drug associated rash in three children: one severe rash, carbamazepine; two mild rash, no drugs stopped. †No clinical hepatitis.

fNine HIV seronegative, two older children not tested.

problem. ${ }^{19}$ The infectiousness and pathogenicity of MDR $M$ tuberculosis strains have been confirmed. ${ }^{5}{ }^{6}$ The emphasis in this study was on culture confirmed childhood MDR TB cases in an area with a high incidence of $\mathrm{TB}$ and a relatively low incidence of MDR TB. ${ }^{15}$ Culture confirmation in children is at best $30-70 \%$, and the need for a positive culture may bias the study population towards younger children or adolescents, and those with more advanced disease, from whom positive cultures are more frequent.

The clinical and radiological presentation of drug susceptible and drug resistant cases is similar. ${ }^{6}{ }^{15}$ Although there is no control group of culture positive drug susceptible TB cases for this study, and compared to previous studies in children from the same area this study group is slightly older (upper range $<15$ years instead of $<13$ years of age), ${ }^{20}$ a relatively high percentage $(36 \%)$ had cavitatory disease on chest radiographs and were smear positive for AFB (44\%). This could be due to disease progression because of the delay in diagnosis, but does imply that children, especially older children, can contribute to the spread of MDR TB. No screening of community contacts was done in this study.

HIV infection was found in $21 \%$ of children who were tested compared to the $13 \%$ infection rate in a previous study of culture positive childhood TB cases done from 1994 to 1998 in the same area. ${ }^{15}$ The higher rate in this study is most likely because of the corresponding rise in HIV infection in this region.

The mortality and morbidity from childhood TB may be influenced by the delay from the time of first symptoms until the start of compliant treatment, ${ }^{9}$ and this delay may further

Table 5 Weight response and chest radiograph results at or near treatment completion

\begin{tabular}{ll}
\hline & No. (\%) \\
\hline $\begin{array}{l}\text { Weight according to NCHS weight for age } \\
\text { percentiles }\end{array}$ & $(\mathrm{n}=33)$ \\
$\begin{array}{l}\text { Weight gain crossing percentiles upwards } \\
\text { Weight following percentiles line }\end{array}$ & $24(73)$ \\
$\begin{array}{l}\text { Weight loss } \\
\text { Follow up chest radiograph results } \\
\text { (last available) }\end{array}$ & $2(21)$ [HIV infected $=1]$ \\
Normal chest radiograph & $(\mathrm{n}=34)$ \\
Calcification only & $15(44)$ \\
Fibrosis only & $5(15)$ \\
Calcification and fibrosis & $5(15)$ \\
Bronchiectasis or destroyed lung & $2(6)$ \\
Lymphoid interstitial pneumonitis & $6(18)$ \\
\hline
\end{tabular}

be prolonged before appropriate treatment is started in the case of MDR TB. The delay from diagnosis of TB to appropriate treatment can be short and similar to that of drug susceptible cases if a history of contact with an infectious case of MDR TB is obtained from the outset (table 2). However, when this history was disregarded or not sought, the delay in initiating MDR TB treatment rose dramatically from a median of 2 days to a median of 17 weeks. In two cases, the extensive delay was probably responsible for the death of these children. In other children disease progression contributed to increased morbidity.

Delay in treatment for MDR TB is often not preventable if no MDR source cases are identified and culture and susceptibility tests are not routine. The highest burden of TB cases is often found in developing countries and, as is the case in South Africa, adult TB cases are routinely identified by sputum smear for AFB only because of a lack of resources. Culture and drug susceptibility testing is reserved for those who do not respond to standard antituberculosis treatment. Cultures of $M$ tuberculosis from children are difficult to obtain, ${ }^{20}$ and when obtained, drug susceptibility tests are often not routinely done because of cost constraints. This can lead to extensive delays in starting appropriate treatment. When MDR TB is suspected because of poor response to treatment, the final results of culture and susceptibility tests needed to identify the correct treatment regimen may not be available for 2-6 months. (table 2). Routine susceptibility testing for all patients and the development of faster susceptibility testing methods would significantly decrease the diagnostic delay.

The possibility of drug resistance should be considered in the management of children if: (a) they have a known adult source case with drug resistant TB; (b) there is no known source case, but the community (or country) in which the child resides (or had resided) has a high prevalence of drug resistant TB; (c) an adult source case is a treatment defaulter (not compliant), a treatment failure (compliant but sputum still positive at end of treatment), a retreatment case (second episode of $\mathrm{TB}$ ), or a chronic case (TB despite two previous treatment courses) with unknown drug susceptibility pattern; (d) a child does not respond satisfactorily or deteriorates while on TB treatment and is compliant; and (e) a child with pulmonary $\mathrm{TB}$ relapses after incomplete or incorrect $\mathrm{TB}$ treatment.

It is usually assumed that children develop TB from recent infection. ${ }^{21}$ Transmission of MDR TB from adult source cases to child contacts has been confirmed by drug susceptibility pattern and RFLP analysis. ${ }^{5}$ According to drug susceptibility results of adult source cases and child contacts, there was a 
high correlation of $68 \%$ between the susceptibility patterns of the MDR TB adult-child pairs. This is similar to the finding of Steiner and colleagues ${ }^{22}$ in a previous study of adult-child transmission of drug resistant TB. If the variability of susceptibility results of the second line drugs is taken into account, this correlation will further be increased..$^{23}$ In one case almost certainly the susceptibility result of the child was incorrectly reported as fully susceptible, while by RFLP analysis she had an identical strain to the mother's who had been a MDR TB case for several years.

In this study $44 \%$ of the children had received previous treatment and, although these cases can be classified as previously treated MDR TB, in the majority of cases reasons could be identified that make transmission of a MDR strain likely. Truly acquired MDR TB was considered likely in mainly a few adolescents who had "adult type" cavitatory disease and failed to comply with initial treatment.

The optimal duration of treatment and drugs that should be used remain uncertain. ${ }^{11}$ INH was used in most regimens because about half of the patients with new INH resistance have low level resistance. ${ }^{15} 24$ Ethambutol has been accepted as a first line TB agent, ${ }^{18}$ and increased resistance to this drug can be expected; $10 \%$ of children had ethambutol resistance.

Ethionamide caused gastrointestinal discomfort in $41 \%$ of patients, which led to discontinuation of the drug in $20 \%$ of cases. These problems can be managed by starting with a lower dose and initially dividing the daily dose. ${ }^{7}$

In this study 32 children received ofloxacin for a median duration of 12 months. Gastrointestinal adverse events occurred in four children, in two of whom the agent was discontinued. Careful scrutiny of the folders revealed no reports of joint pain in any of these children. It was disconcerting that two children were infected with ofloxacin resistant strains.

The outcome of MDR TB in adults has been notoriously poor. Mortality rates have been especially high in HIV infected MDR cases, but mortality rates of $39-48 \%$ have been reported in HIV seronegative MDR TB cases. ${ }^{13}{ }^{14}$ The known mortality in this study who all presented with clinical disease, was 10\%; a further two children were lost to follow up without any treatment. Of the four known deaths, MDR TB was not the cause in one. Earlier recognition of drug resistance could probably have prevented a further two deaths and morbidity. Of those children who were followed up after the completion of treatment, none had a relapse of MDR TB.

This study illustrates the potentially serious consequences of a delay in appropriate treatment for MDR TB and the importance of a detailed contact history. Furthermore, where the resources are available drug susceptibility should preferably be determined in all children with culture positive TB.

\section{ACKNOWLEDGEMENTS}

The authors would like to thank the Harry and Doris Crossley Foundation for financial assistance and the clinic nursing staff at the Western Cape local authority health clinics.

\section{Authors' affiliations}

H S Schaaf, P R Donald, Department of Paediatrics and Child Health, Faculty of Health Sciences, Stellenbosch University and Tygerberg Children's Hospital, South Africa

K Shean, Brooklyn Hospital for Chest Diseases, Western Cape Province, South Africa

\section{REFERENCES}

1 World Health Organisation. Guidelines for the management of drug-resistant tuberculosis. WHO/TB/96.210 (Rev1). Geneva: WHO, 1997.

2 Iseman MD. Treatment of multidrug-resistant tuberculosis. N Engl J Med 1993;329:784-91.

3 Iseman MD. Treatment and implications of multidrug-resistant tuberculosis for the 21 st century. Chemotherapy 1999;45(suppl 2):34-40.

4 Van Rie A, Warren R, Richardson M, et al. Classification of drug-resistant tuberculosis in an epidemic area. Lancet 2000;356:22-5

5 Schaaf HS, Van Rie A, Gie RP, et al. Transmission of multidrug resistant tuberculosis. Pediatr Infect Dis J 2000; 19:695-9.

6 Snider DE Jr, Kelly GD, Cauthen GM, et al. Infection and disease among contacts of tuberculosis cases with drug-resistant and drug-susceptible bacilli. Am Rev Respir Dis 1985;132:125-32.

7 Schaaf HS, Gie RP, Kennedy M, et al. Evaluation of young children in contact with adult multidrug resistant pulmonary tuberculosis: a 30-month follow-up. Pediatrics 2002;109:765-71.

8 Schauf HS, Vermeulen HAS, Gie RP, et al. Evaluation of young children in household contact with adult multidrug-resistant pulmonary tuberculosis cases. Pediatr Infect Dis J 1999:18:494-500.

9 Beyers N, Gie RP, Schaaf HS, et al. Delay in the diagnosis, notification and initiation of treatment and compliance in children with tuberculosis. Tuberc Lung Dis 1994;75:260-5.

10 Iseman MD, Madsen LA. Drug-resistant tuberculosis. Clin Chest Med 1989; 10:341-53.

11 Swanson DS, Starke JR. Drug-resistant tuberculosis in pediatrics. Pediatr Clin N Am 1995;42:553-81.

12 Schaad UB. Pediatric use of quinolones. Pediatr Infect Dis J 1999; 18:469-70.

13 Schaaf HS, Botha P, Beyers N, et al. The 5-year outcome of multidrug resistant tuberculosis patients in the Cape Province of South Africa. Trop Med Int Health 1996;1:718-22.

14 Goble M, Iseman MD, Madsen LD, et al. Treatment of 171 patients with pulmonary tuberculosis resistant to isoniazid and rifampin. N Engl J Med 1993;328:527-32.

15 Schaaf HS, Gie RP, Beyers N, et al. Primary drug-resistant tuberculosis in children. Int J Tuberc Lung Dis 2000;12:1149-55.

16 Harries AD, Maher D. Diagnosis of tuberculosis in children. In: TB/HIV: a clinical manual. WHO/TB/96.200. Geneva: WHO, 1996:61-8.

17 Smuts NA, Beyers N, Gie RP, et al. Value of the lateral chest radiograph in tuberculosis in children. Pediatr Radiol 1994;24:478-80.

18 World Health Organisation. Treatment of tuberculosis: guidelines for national programmes, 2nd edn. WHO/TB/97.220. Geneva: WHO, 1997.

19 Espinal MA, Laszlo A, Simonsen L, et al. Global trends in resistance to antituberculosis drugs. N Engl J Med 2001;344:1294-303.

20 Schaaf HS, Beyers N, Gie RP, et al. Respiratory tuberculosis in childhood: the diagnostic value of clinical features and special investigations. Pediatr Infect Dis J 1995; 14:189-94.

21 Rieder HL. Drug-resistant tuberculosis: issues in epidemiology and challenges for public health. Tuberc Lung Dis 1993;75:321-3.

22 Steiner $\mathbf{P}$, Rao M, Mitchell M, et al. Primary drug-resistant tuberculosis in children: correlation of drug-susceptibility patterns of matched patient and source case strains of Mycobacterium tuberculosis. Am J Dis Child $1985 ; 139: 780-2$.

23 Laszlo A. How accurate is drug susceptibility testing for second-line drugs: do we need a proficiency testing exercise? Int J Tuberc Lung Dis 2002;6/suppl 1):S29.

24 Tripathy SP, Menon NK, Mitchison DA, et al. Response to treatment with isoniazid plus PAS of tuberculosis patients with primary isoniazid resistance. Tubercle 1969;50:257-68. 